\title{
RÖKO DIGITAL - Angebot für MTRA-Schulen
}

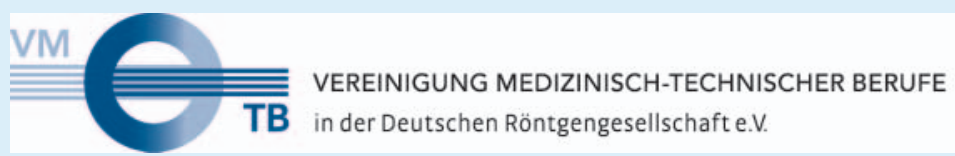

Das MTRA-Schülerprogramm war in den vergangenen Jahren fester Bestandteil des Deutschen Röntgenkongresses. Die Präsenzveranstaltung kann COVID-bedingt in Leipzig leider nicht mehr stattfinden. Stattdessen wird der Kongress als RÖKO DIGITAL durchgeführt.

Sowohl das MTRA-Programm als auch die ärztlichen Fortbildungen des RÖKO DIGITAL werden aufgezeichnet. Die VMTB möchte diese Aufzeichnungen gerne den MTRASchulen für ihren Unterricht auf conrad, der digitalen Lernplattform der DRG, zur Verfügung stellen. Angemeldete Schulen erhalten dann einen Zugangslink und können diesen allen MTRA-Schülerinnen und -Schülern sowie den Lehrkräften ihrer Einrichtung zur Verfügung stellen. Die Anmeldung kostet einmalig $250 €$ pro Schule. Der Zugangslink ist bis Ende des Jahres gültig. Sie haben dann Zugriff auf alle MTRA-Vorträge (Auf- zeichnungen von 12 Terminen) und auf die Highlight-Sessions des RÖKO DIGITAL. Die Aufzeichnungen werden im Laufe des Jahres in conrad eingestellt (in der Regel innerhalb einer Woche nach der Live-Veranstaltung) und Ihnen zur Verfügung gestellt.

\section{SIE MÖCHTEN IHRE SCHULE ANMELDEN?}

Dann nutzen Sie das Anmeldeformular auf www.vmtb.de > Über uns > Meldungen 